\title{
Adjustable Inertial Response from the Converter with Adaptive Droop Control in DC Grids
}

\author{
Yi Wang, Member, IEEE, Chen Wang, Lie Xu, Senior Member, IEEE, Jianhui Meng, and Yang Hei
}

\begin{abstract}
In a DC grid, the inherent inertial support from the DC capacitors is too small to resist step changes or random fluctuations from the intermittent power resources, which results in lower DC voltage quality. In this paper, an adaptive droop control (ADC) strategy is proposed to achieve an increased inertia from the droop controlled converter. The adaptable droop coefficient according to the DC voltage variation enables fast swing of the droop curve, so that the converter can provide inertial power for the DC grid like synchronous generators in AC grids. The design of the ADC including the calculation and limitation of the adaptable droop coefficient is analyzed in detail. The smallsignal analysis of the DC grid with ADC is provided to identify its stability issue. Experimental tests on a controller hardware-in-theloop (HIL) platform of a low-voltage (LV) DC grid are carried out to validate the proposed method. In this LV DC grid, the proposed ADC is implemented on the energy storage system (ESS) which provides inertial support to improve the DC voltage quality under different power fluctuations, and smooths the power transmitted to AC grid.
\end{abstract}

Index Terms - DC grid, virtual inertia, voltage droop control, variable droop coefficient, adaptive control.

\section{INTRODUCTION}

$\mathrm{D}^{\mathrm{c}}$ grids are currently considered as creditable alternatives to AC grids for integrating renewable energy sources (RES) due to the distinct advantages, such as less power conversion and transmission loss, no concerns on reactive power consumption and power angle stability, and flexible power flow controllability [1]-[2]. However, the capacitance inertia of DC grids is far less than the mechanic inertia of conventional AC grids with synchronous generators [3]-[5]. During large disturbances, the abrupt change of the DC bus voltage may give rise to harmful impacts on sensitive loads and RES units. Moreover, step changes and random fluctuations from the intermittent generations can lead to drastic fluctuations of the DC bus voltage and power transmitted to AC grid. The voltage droop control is utilized on the converters in the DC grid to ensure its voltage stability by redistributing power automatically during disturbances [6]-[7]. However, the output power of the converter has to approach a new operating point by following the droop curve during disturbances. Thus, it cannot mitigate the mutation rate of the DC voltage effectively due to the distinct power unbalance on the capacitor exits.

This work was supported by the National Key R\&D Program of China under Grants 2016YFB0900203.

Y. Wang, C. Wang, J. Meng and Y. Hei are with the State Key Laboratory of Alternate Electrical Power System with Renewable Energy Sources, North China Electric Power University, Baoding 071003, China (e-mail: yi.wang@ncepu.edu.cn).

L. Xu is with the Dept. of Electronic \& Electrical Engineering, University of Strathclyde, Glasgow, UK.
Therefore, introducing virtual inertia control to the DC grid is necessary to improve its voltage quality.

The AC grid with high penetration rate of RES is also a lowinertia system [8]-[9]. To resolve this problem, virtual inertia control methods arouse great attention in recent years. The concept of virtual synchronous machines (VSM) is proposed in [10] and the moment of inertia in synchronous machines can be emulated by the converters of RES. In [11] and [12], inverters named synchronverters are controlled to behave in the same way as synchronous generators to ensure smooth transition of inverter dominated grids. In [13], an optimized power point tracking (OPPT) controller is proposed to shift the operating point of a permanent magnet synchronous generator (PMSG)based wind turbine from the MPPT curve to the virtual inertia control curve according to the frequency deviation.

In DC grids, the DC capacitors store energy and create a kind of inertia for DC voltage to resist its rapid change. However, the equivalent inertia of the capacitors is far less than that of the rotating mass of a synchronous generator with similar VA rating. Therefore, the DC grid is a small inertia system and its DC voltage can vary rapidly during power imbalance. The virtual inertia control can be introduced to the DC grid to prevent fast DC voltage transient, though there has been limited research on the topic. In [14], an ultra-capacitor is added to offer a transient compensation when a sudden load is switched on and therefore the transient response is improved. However, the ultra-capacitor has high cost and remains idle when the DC grid is under steady-state operation, which makes it unproductive. To avoid the over-charging and over-discharging conditions for the energy storage system (ESS), virtual inertia control is proposed for the RES units in a DC grid [15]. However, it mainly focuses on the coordinated control among microsources with little details on the analysis of the virtual inertia control. In [16], the DC bus voltage is introduced to the speed regulation of PMSG-based wind turbines and a virtual inertia control strategy for the wind-battery-based islanded DC microgrid is proposed. The rotational kinetic energy of the wind turbines is utilized to supply virtual inertia, but it is not suitable for other kind of RES such as the PV generation. In [17], a virtual inertia control strategy analogized with VSM is presented to enhance the inertia of the DC microgrid. However, an auxiliary control loop to emulate VSM characteristic has to be added in the converter control system. Therefore, for droop controlled converters in DC grids, the coordination between supplemental inertial control and primary voltage regulation needs to be considered.

Voltage droop control is regarded as a practical scheme for power sharing in DC grids [18]-[21]. Some modified droop 
controllers have been presented recently to further enhance the voltage quality and stability of DC grids [22-27]. In [22], an improved decentralized DC power management strategy based on $P-V_{\mathrm{dc}}{ }^{2}$ droop is designed to enhance the dynamics of DC power regulation. In [23], a droop controller suitable for multitime scale power control is presented to realize proper power interaction among different sub-grids. In [24], the relationship between AC frequency and DC voltage identified via the interactive virtual inertia and capacitance is used to design the voltage control strategy for AC/DC interface for improving the system stability. In [25], an observer-based DC voltage droop and a current feed-forward control for a DC microgrid are proposed to improve the dynamic response of DC voltage control. Although abovementioned control schemes can improve the dynamic performances of DC grids, the controller designs tend to be more complicated than the conventional droop control, due to the fact that modified control for dynamics improvement may be coupled with the primary voltage regulation and power sharing.

This paper proposes a control method to combine the virtual inertia control with the voltage droop control to increase the inertia of DC grids without additional controllers. The proposed adaptive droop control (ADC) will initially utilize a fast swing of the droop curve to emulate inertial response as a virtual capacitance during disturbances, whereas the droop curve will resume fixed as the DC voltage approaches to a steady state. Thus, the swing of the droop curve only takes effect to suppress the voltage mutation at the initial stage of disturbances, and has little effect on the subsequent primary voltage regulation and power sharing. Therefore, the inertial response obtained by this way can be more easily integrated with the conventional droop control. This paper is organized as follows. Section II illustrates the DC grid under investigation and gives the principle of the voltage droop control. Detailed inertia analysis of the DC grid is described in Section III, and the study of the virtual inertia control based on the variable droop coefficient is carried out. By modifying the variable droop coefficient, an adaptive droop control strategy with inertial response is proposed in Section IV. Section $\mathrm{V}$ investigates the stability issue of the DC grid with ADC using small-signal analysis. Experimental results based on a hardware-in-the-loop (HIL) platform of a low-voltage (LV) DC grid are presented in Section VI to validate the proposed control strategy. Finally, conclusions are drawn in Section VII.

\section{DC GRID CONFIGURATION AND CONTROL}

\section{A. Configuration of the DC Grid for RES Integration}

A typical configuration of the DC grid for RES integration is shown in Fig. 1, where four different kinds of terminals are connected to the DC grid by converters, i.e. RES, AC/DC loads, AC grid and energy storage system (ESS). The DC transformers (DC-DC converter), namely P-DCT and W-DCT, are used to connect the PV units and wind turbines to the DC grid, respectively. AC loads are connected to the DC bus via a DCAC converter L-VSC, while DC loads can be connected to the DC bus directly or via a DC transformer L-DCT. The G-VSC connects the DC grid to the AC grid. The ESS of a DC grid can be based on batteries, super capacitors, flywheels, etc. Battery energy storage system (BESS) is most commonly used and is discussed in this paper. The BESS is connected to the DC bus via a bidirectional DC transformer E-DCT.
The RES units and loads usually operate on their own merits and do not actively contribute to system control. The G-VSC and BESS are responsible for balancing the power surplus/deficit caused by RES units and loads and ensuring stable operation of the system. In this paper, voltage droop control is adopted as the primary voltage regulation strategy for the DC grid to coordinate the G-VSC and E-DCT (BESS).

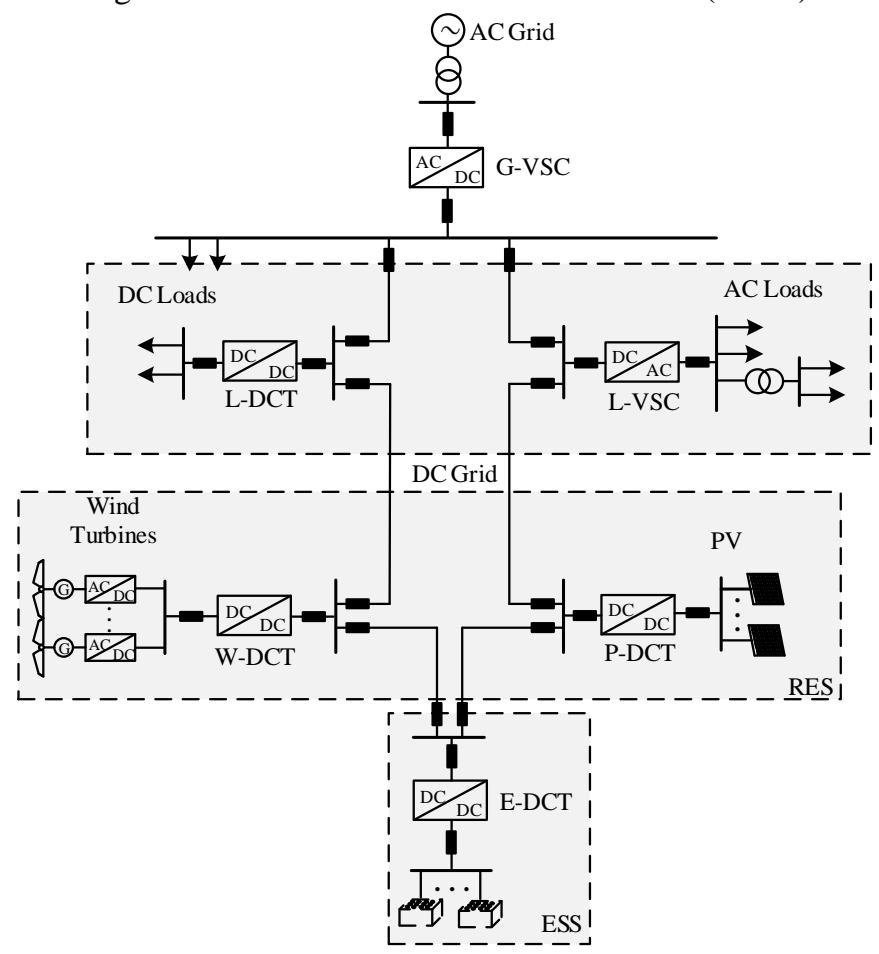

Fig. 1. Typical configuration of a DC grid for RES integration.

\section{B. Voltage Droop Control}

One of the classical decentralized power management methods is the voltage-power $(V-P)$ droop control, in which the deviation of the DC bus voltage is used for autonomous power sharing among different sources. The characteristics of $V-P$ droop control are shown in Fig. 2, which can be expressed as

$$
V_{\mathrm{dc}}=V_{\mathrm{dc}}^{*}-\frac{1}{k}\left(P-P^{*}\right)
$$

where $V_{\mathrm{dc}}$ and $P$ are the DC bus voltage and output active power of the converter respectively. The superscript $*$ refers to the reference values, and $k$ is the droop coefficient.

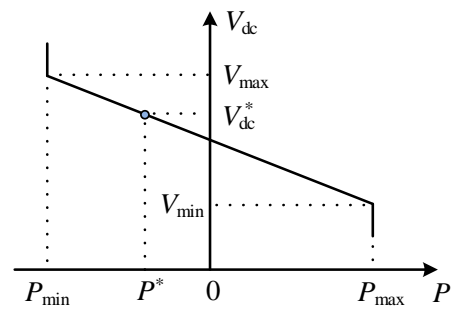

Fig. 2. Characteristics of $V-P$ droop control.

\section{PRINCIPLE OF ADAPTIVE DROOP CONTROL WITH ADJUSTABLE INERTIA}

For a DC grid using droop control, only capacitors directly contribute to the resistance of the voltage change rate $\mathrm{d} V_{\mathrm{dc}} / \mathrm{d} t$. This can lead to the deterioration of DC voltage quality, such as 
voltage flicker and higher-frequency fluctuations. In this section, DC grid inertia is introduced and detailed analysis of the controllable inertia of the DC grid based on adaptive droop control is described in order to reduce the transient DC voltage variation.

\section{A. DC Grid Inertia Definition}

For an AC power system, inertia reflects the system capability in preventing the frequency from a sudden change. Due to the existence of inertia, generators have sufficient time to adjust their generated power and reestablish the power balance. The inertia time constant $H_{\mathrm{s}}$ of a traditional synchronous generator can be defined as

$$
H_{\mathrm{s}}=\frac{W_{\mathrm{k}}}{S_{\mathrm{N}}}
$$

where $W_{\mathrm{k}}$ is the kinetic energy stored in the rotor at rated rotating speed and $S_{\mathrm{N}}$ is the nominal generation capacity.

The definition of the DC grid inertia can be developed from the same concept of the AC system, i.e. DC power system inertia reflects the system capability in preventing the DC voltage from a sudden change. Generalizing (2) to DC systems, the inertia time constant $H_{\mathrm{dc}}$ of one terminal of the DC grid can be defined as

$$
H_{\mathrm{dc}}=\frac{W_{\mathrm{e}}}{S_{\mathrm{Nc}}}=\frac{C}{2} \frac{V_{\mathrm{N}}^{2}}{S_{\mathrm{Nc}}}
$$

where $W_{\mathrm{e}}$ and $S_{\mathrm{Nc}}$ refer to the stored capacitor energy at nominal DC voltage $V_{\mathrm{N}}$ and nominal capacity, respectively. $C$ is the capacitance value of a DC terminal. The physical meaning of $H_{\mathrm{dc}}$ can be described as the amount of time required to release all the electric energy stored in the capacitor at the rate of $S_{\mathrm{Nc}}$. Therefore, the larger $C$ is, the larger $H_{\mathrm{dc}}$ and DC grid inertia are.

For a synchronous generator, the typical value of $H_{\mathrm{s}}$ is approximately a few seconds. For a DC terminal, according to (3) and assuming the rated $V_{\mathrm{N}}$ and $S_{\mathrm{Nc}}$ of $10 \mathrm{kV}$ and $4 \mathrm{MW}$, a capacitor with a few tenth of farad is required to make $H_{\mathrm{dc}}$ possess the same magnitude order as $H_{\mathrm{s}}$. However, the required $C$ value is much larger than that of a typical DC capacitor in such a system.

\section{B. Principle of Virtual Inertia in DC Grid}

The equivalent circuit of a converter in DC grids is shown in Fig. 3(a), where $P_{\mathrm{R}}$ represents the total power by the RES units and loads, and $P_{\mathrm{S}}$ represents the power supplied by a power source such as the BESS. The power relationship of the circuit in Fig. 3(a) can be expressed as

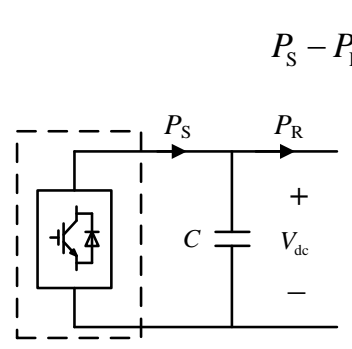

(a)

$$
P_{\mathrm{S}}-P_{\mathrm{R}}=C V_{\mathrm{dc}} \frac{d V_{\mathrm{dc}}}{d t} \text {. }
$$

Fig. 3. Equivalent circuit of a converter in DC grids. (a) Without virtual inertia control. (b) With virtual inertia control.

It can be inferred from (4) that a constant DC voltage indicates a balanced power flow. During system disturbances such as power fluctuations by the RES units and/or loads, the larger $C$ is, the smaller $d V_{\mathrm{dc}} / d t$ becomes. However, as analyzed above, the DC capacitance value is small in practice.

In order to increase the equivalent inertia of a DC system, virtual inertia control is adopted and is implemented on the converters. As shown in Fig. 3(b), the auxiliary power $\Delta P_{\mathrm{S}}$ is supplied to slow down the voltage change rate during the variation of DC bus voltage as given by

$$
\Delta P_{\mathrm{S}}=C_{\mathrm{vir}} V_{\mathrm{dc}} \frac{d V_{\mathrm{dc}}}{d t}
$$

where $C_{\text {vir }}$ is the equivalent capacitance added by virtual inertia control in the DC side of the converter, referred to as the virtual capacitance. Taking $\Delta P_{\mathrm{S}}$ into account, the supplied power can be updated as

$$
P_{\mathrm{S}}=P_{\mathrm{S}}^{\prime}-\Delta P_{\mathrm{S}}
$$

where $P_{\mathrm{S}}^{\prime}$ is the supplied power without virtual inertia control.

Substituting (5) and (6) into (4) yields

$$
\left(C+C_{\mathrm{vir}}\right) V_{\mathrm{dc}} \frac{d V_{\mathrm{dc}}}{d t}=P_{\mathrm{S}}^{\prime}-P_{\mathrm{R}} .
$$

Equation (7) indicates that during the variation of $V_{\mathrm{dc}}$, the equivalent DC capacitance is enlarged because of $C_{\mathrm{vir}}$ by the virtual inertia control. According to (3) and taking $C_{\text {vir }}$ into account, $H_{\mathrm{dc}}$ can be modified as

$$
H_{\mathrm{dc}}=\frac{C+C_{\mathrm{vir}}}{2} \frac{V_{\mathrm{N}}^{2}}{S_{\mathrm{Nc}}} .
$$

From (8), it can be seen that with the virtual inertia control, the inertia of the DC grid is increased and a reduced $d V_{\mathrm{dc}} / d t$ can be obtained.

\section{Virtual Inertial Response by Swing of Droop Curve}

The auxiliary power mentioned above can be supplied quickly by swinging the voltage droop curve and the dynamic swing of the droop curve can be realized by changing the droop coefficient. Figs. 4 (a) and (b) show the relevant schematic diagrams for positive (supply) and negative (absorb) power directions, respectively, where $\mathrm{O}$ is the reference point $\left(P^{*}\right.$ is normally taken as 0 ) and $\mathrm{A}$ is the steady state operating point on the droop curve (1). $V_{\mathrm{A}}$ and $P_{\mathrm{A}}$ refer to the corresponding DC voltage and active power of point $A$, respectively. Under steady state, $P_{\mathrm{R}}=P_{\mathrm{S}}=P_{\mathrm{A}}$.

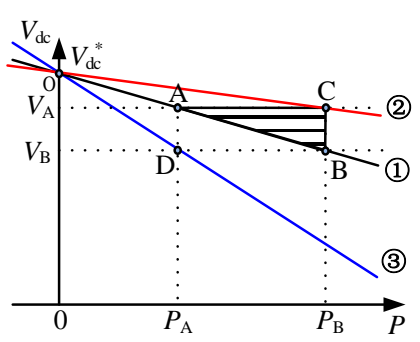

(a)

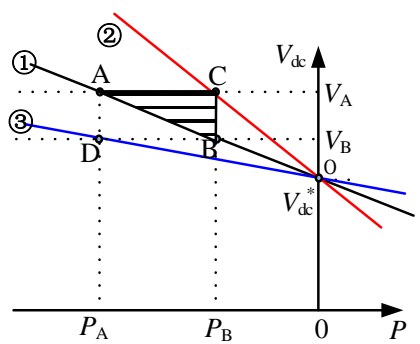

(b)
Fig. 4. Schematic diagram of virtual inertial response by swing of droop control curve. (a) $P>0$. (b) $P<0$.

When $P_{\mathrm{R}}$ suddenly increased from $P_{\mathrm{A}}$ to $P_{\mathrm{B}}$, the unbalanced power between $P_{\mathrm{R}}$ and $P_{\mathrm{S}}$ during this transient process causes a rapid decline of the capacitor voltage (i.e. $d V_{\mathrm{dc}} / d t<0$ ). The operating point of the converter will move from $\mathrm{A}$ to $\mathrm{B}$ along the curve (1) to increase its power output from $P_{\mathrm{A}}$ to $P_{\mathrm{B}}$ under 
the normal droop control. The power difference between $P_{\mathrm{R}}$ and $P_{\mathrm{S}}$ during this transient process will be reduced from $P_{\mathrm{B}}-P_{\mathrm{A}}$ to 0 along the curve $\mathrm{AB}$ gradually. To provide virtual inertia response, if $P>0$, the droop curve quickly swings anti-clockwise form (1) to (2) by increasing the droop coefficient $k$, as shown in Fig. 4(a). If $P<0$, curve (1) swings clockwise to curve (2) by decreasing $k$, as shown in Fig. 4(b). Thus, the converter can immediately release power from $P_{\mathrm{A}}$ to $P_{\mathrm{B}}$ without large DC voltage drop. When a suddenly reduction of $P_{\mathrm{R}}$ from $P_{\mathrm{B}}$ to $P_{\mathrm{A}}$ occurs (i.e. $d V_{\mathrm{dc}} / d t>0$ ), curve (1) swings clockwise/anticlockwise to curve (3) by decreasing/increasing $k$ and at the same time, the converter can absorb the power quickly to slow down the change rate of the capacitor voltage.

According to the above analysis, the fast swing of the droop control curve can make the converter release/absorb power more quickly than the fixed droop curve, so as to achieve the inertial response of resisting the voltage change rate. The variable droop coefficient $k$ can be associated with $d V_{\mathrm{dc}} / d t$ and is defined as

$$
\begin{gathered}
k=K_{1}+\operatorname{sign}\left(V_{\mathrm{dc}}-V_{\mathrm{dc}}^{*}\right) \cdot K_{2} \frac{d V_{\mathrm{dc}}}{d t}=K_{1}+K_{2}^{\prime} \frac{d V_{\mathrm{dc}}}{d t} \\
K_{2}^{\prime}=\operatorname{sign}\left(V_{\mathrm{dc}}-V_{\mathrm{dc}}^{*}\right) \cdot K_{2}
\end{gathered}
$$

where $K_{1}$ is the droop coefficient under steady state. $K_{2}$ is the virtual inertia coefficient, and $K_{2}>0$.

As seen in (9) and (10), $k$ is proportional to $d V_{\mathrm{dc}} / d t$ and the sign symbol of $K_{2}^{\prime}$ is determined by the position relationship between the operating point and the reference point. Under steady state, $d V_{\mathrm{dc}} / d t$ is equal to zero and $k$ remains constant at $K_{1}$. Once $V_{\mathrm{dc}}$ varies due to power disturbances, $k$ increases or decreases and the droop curve swings to offer auxiliary power to enhance the inertia of the DC system. For purpose of preventing the absorbed power from exceeding the maximum value, swing range of the droop curve should be limited between the lower limit $K_{\min }$ and the upper limit $K_{\max }$.

In order to estimate the virtual inertia supplied by virtual inertia control based on the variable droop coefficient given in (9), the virtual capacitance $C_{\text {vir }}$ is deduced here.

Substituting (9) into (1) and according to (6), $P_{\mathrm{S}}$ is obtained as

$$
\begin{aligned}
P_{\mathrm{S}} & =K_{1}\left(V_{\mathrm{dc}}^{*}-V_{\mathrm{dc}}\right)+K_{2}^{\prime}\left(V_{\mathrm{dc}}^{*}-V_{\mathrm{dc}}\right) \frac{d V_{\mathrm{dc}}}{d t} . \\
& =P_{\mathrm{S}}^{\prime}+K_{2}^{\prime}\left(V_{\mathrm{dc}}^{*}-V_{\mathrm{dc}}\right) \frac{d V_{\mathrm{dc}}}{d t}
\end{aligned}
$$

Substituting (11) into (4) yields

$$
C V_{\mathrm{dc}} \frac{d V_{\mathrm{dc}}}{d t}+K_{2}^{\prime}\left(V_{\mathrm{dc}}-V_{\mathrm{dc}}^{*}\right) \frac{d V_{\mathrm{dc}}}{d t}=P_{\mathrm{S}}^{\prime}-P_{\mathrm{R}} .
$$

Comparing (12) with (7), $C_{\text {vir }}$ can be obtained as

$$
C_{\mathrm{vir}}=K_{2}^{\prime}\left(\frac{V_{\mathrm{dc}}-V_{\mathrm{dc}}^{*}}{V_{\mathrm{dc}}}\right)=K_{2} \frac{\left|V_{\mathrm{dc}}-V_{\mathrm{dc}}^{*}\right|}{V_{\mathrm{dc}}} .
$$

Equation (13) reveals that $C_{\mathrm{vir}}$ varies with $V_{\mathrm{dc}}$ and is controllable by adjusting $K_{2}$. Thus, the virtual inertia emulated by the adaptive droop control is not fixed, and it can be adjusted as required. The larger $K_{2}$ is, the larger $C_{\text {vir }}$ and supplied virtual inertia are. After the inertial support by fast swing of the droop curve, the droop coefficient will recover to its original value with the voltage gradually stabilized.

\section{DESIGN OF ADAPTIVE DROOP CONTROL STRATEGY}

Within the limited swing range, the faster swing speed of the droop curve are, the larger supplied virtual inertia becomes. To balance the inertia response speed (i.e. improve the swing speed of the droop curve by a large $K_{2}$ ) and system stability, and to take $K_{\min }$ and $K_{\max }$ into account, the arc-tangent function for droop coefficient calculation is employed here. The design of this ADC strategy with controllable inertia is presented as follows.

\section{A. Improved Adaptive Droop Coefficient Calculation}

To realize adaptive swing of the droop curve within the allowable range, the arc-tangent function is adopted to improve the variable droop coefficient $k$ given in (9). In view of the above analysis on Fig. 4 , when $P<0$, the improved $k$ considering $K_{\min }$ and $K_{\max }$ can be given as

$$
k=\left\{\begin{array}{lc}
K_{1}+\frac{K_{\mathrm{max}}-K_{1}}{\pi / 2} \arctan \left(K_{2}^{\prime} \frac{d V_{\mathrm{dc}}}{d t}\right) & \frac{d V_{\mathrm{dc}}}{d t}>0 \\
K_{1}+\frac{K_{1}-K_{\min }}{\pi / 2} \arctan \left(K_{2}^{\prime} \frac{d V_{\mathrm{dc}}}{d t}\right) & \frac{d V_{\mathrm{dc}}}{d t}<0
\end{array} .\right.
$$

When $P>0$, the improved $k$ can be given as

$$
k=\left\{\begin{array}{ll}
K_{1}+\frac{K_{1}-K_{\min }}{\pi / 2} \arctan \left(K_{2}^{\prime} \frac{d V_{\mathrm{dc}}}{d t}\right) & \frac{d V_{\mathrm{dc}}}{d t}>0 \\
K_{1}+\frac{K_{\max }-K_{1}}{\pi / 2} \arctan \left(K_{2}^{\prime} \frac{d V_{\mathrm{dc}}}{d t}\right) & \frac{d V_{\mathrm{dc}}}{d t}<0
\end{array} .\right.
$$

Combining (14) and (15) and taking the sign of $K_{2}^{\prime}$ into account, the improved $k$ can be given as

$$
k=\left\{\begin{array}{ll}
K_{1}+\frac{K_{\mathrm{max}}-K_{1}}{\pi / 2} \arctan \left(K_{2}^{\prime} \frac{d V_{\mathrm{dc}}}{d t}\right) & K_{2}^{\prime} \frac{d V_{\mathrm{dc}}}{d t}>0 \\
K_{1}+\frac{K_{1}-K_{\min }}{\pi / 2} \arctan \left(K_{2}^{\prime} \frac{d V_{\mathrm{dc}}}{d t}\right) & K_{2}^{\prime} \frac{d V_{\mathrm{dc}}}{d t}<0
\end{array} .\right.
$$

In a practical control system, the detection of $d V_{\mathrm{dc}} / d t$ is sensitive to noise and harmonic interference. Considering practical implementation, a washout filter is adopted to achieve the transient voltage variation $\delta V_{\mathrm{dc}}$ instead of $d V_{\mathrm{dc}} / d t$, and (16) can be modified as

$$
k=\left\{\begin{array}{ll}
K_{1}+\frac{K_{\mathrm{max}}-K_{1}}{\pi / 2} \arctan \left(K_{2}^{\prime} \delta V_{\mathrm{dc}}\right) & K_{2}^{\prime} \delta V_{\mathrm{dc}}>0 \\
K_{1}+\frac{K_{1}-K_{\min }}{\pi / 2} \arctan \left(K_{2}^{\prime} \delta V_{\mathrm{dc}}\right) & K_{2}^{\prime} \delta V_{\mathrm{dc}}<0
\end{array} .\right.
$$

Fig. 5 shows the difference of the calculated droop coefficient by (9) and (16). As seen, the arc-tangent function has a larger slope initially which then reduces gradually. Thus, it is more sensitive to small values and can smoothly transit to the limits. As $\delta V_{\mathrm{dc}}$ is normally smaller than $d V_{\mathrm{dc}} / d t$ especially at the initial stage, the droop coefficient $k$ calculated by arctangent function is more effective. Even for the initial small $\delta V_{\mathrm{dc}}$, the $k$ calculated by (17) can be relatively large, which implies that the swing speed of the droop curve is faster at the initial stage of disturbances. As $\delta V_{\mathrm{dc}}$ further increases, the droop coefficient $k$ will smoothly move to the limits, which is favorable for ensuring voltage stability. 


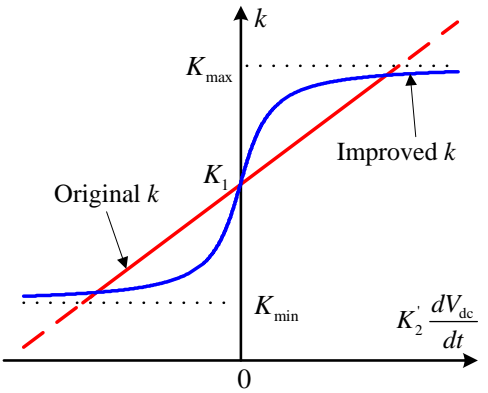

Fig. 5. Comparison between two calculation methods of the variable droop coefficient.

\section{B. Real-Time Calculation of the Limits}

The maximum swing amplitude of the droop curve depends on the limits of the droop coefficient. In order to make the most of the capability of a droop controlled converter in supplying virtual inertia, the real-time calculation of $K_{\max }$ should be executed.

Taking the operating points higher than the reference point (i.e. $P<0$ ) as an example, Fig. 6 compares different limits of $k$ corresponding to three different steady state operating points A, $\mathrm{B}$ and $\mathrm{C}$ on the droop curve (1). For point $\mathrm{A}$, when a power surplus occurs, the converter needs to reduce its power (i.e. further to the negative direction) and thus, the curve (1) can swing anti-clockwise to curve (2) at most with the output power decreasing from $P_{\mathrm{A}}$ to $P_{\min }$ to provide the maximum inertial support. The value of $K_{\max }$ when operating at point A is $K_{\mathrm{A} \_\max }$ and similarly, values of $K_{\max }$ for operating points B and $\mathrm{C}$ are $K_{\mathrm{B} \_\max }$ and $K_{\mathrm{C} \_\max }$, respectively. If the value of $K_{\max }$ for point A is selected as $K_{\mathrm{B} \_ \text {max }}$, curve (1) can only be swung to curve (3) and the power decreases to $P_{\mathrm{A}}^{\prime}$ rather than $P_{\min }$. Since $\left(P_{\mathrm{A}}-P_{\min }\right)$ is the maximum available power for the operating point $\mathrm{A}$, the use of the limit $K_{\mathrm{A}_{\_} \max }$ already ensures it being fully utilized. If $K_{\mathrm{C} \_\max }$ is selected, the curve (1) will swing to curve (4), but no extra power can be output than that using $K_{\mathrm{A} \_\max }$ due to the converter power limit of $P_{\min }$. However, the recovery progress becomes longer for the extra swing range, especially when $P_{\mathrm{A}}$ is close to $P_{\min }$, which is detrimental to suppressing of the high frequency fluctuations. Hence, values of $K_{\max }$ for different operating points should be calculated in real time depending on the operating point prior to the transient. For the operating point being higher than the reference point and anti-clockwise swing, it can move to $\left(P_{\min }, V_{\mathrm{dc}}\right)$ at most. Substituting $\left(P_{\min }, V_{\mathrm{dc}}\right)$ into (1), $K_{\max }$ can be calculated as

$$
K_{\max }=\frac{-P_{\min }}{V_{\mathrm{dc}}-V_{\mathrm{dc}}^{*}} .
$$

The calculation method of $K_{\max }$ for operating points lower than the reference point is similar to that for higher operating points. Taking the position of operating points into account, $K_{\max }$ can be calculated as

$$
K_{\max }=\left\{\begin{array}{ll}
\frac{-P_{\min }}{V_{\mathrm{dc}}-V_{\mathrm{dc}}^{*}} & V_{\mathrm{dc}}>V_{\mathrm{dc}}^{*} \\
\frac{P_{\max }}{V_{\mathrm{dc}}^{*}-V_{\mathrm{dc}}} & V_{\mathrm{dc}}<V_{\mathrm{dc}}^{*}
\end{array} .\right.
$$

According to (19), the values of $K_{\max }$ varies with the moving of the operating point for fully utilizing the available power of the converter. For example, for a power surplus occurring at point A $\left(V_{\mathrm{A}}, P_{\mathrm{A}}\right)$, the yielded point $\left(V_{\mathrm{A}}, P_{\min }\right)$ by substituting (18) to (1) is exactly the maximum power point which point A can be shifted to. Thus, the real-time calculation of $K_{\max }$ can ensure the converter to provide appropriate inertial support under different operating points. Considering that the negative droop coefficient may cause stability issues, $K_{\min }$ in this paper is set to zero.

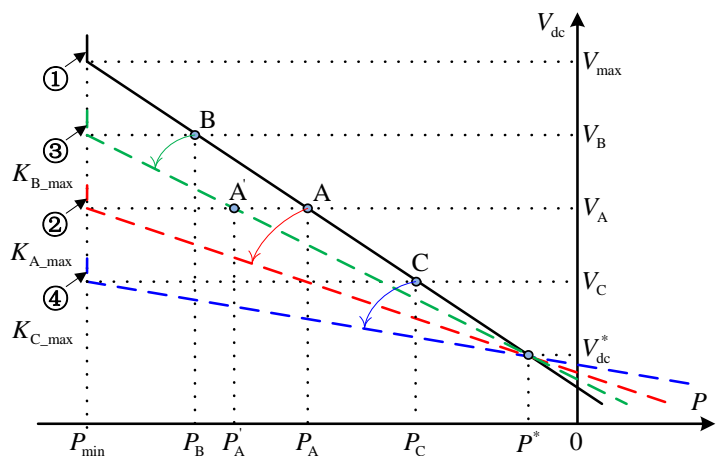

Fig. 6. Different values of $K_{\max }$ for different operating points.

\section{Virtual Inertia Responses with Different Values of $K_{1}$}

Defined as the steady-state droop coefficient, $K_{1}$ reflects the primary voltage regulation ability of the converter and can be set accordingly though different $K_{1}$ values can cause difference on the virtual inertia supplied by ADC.

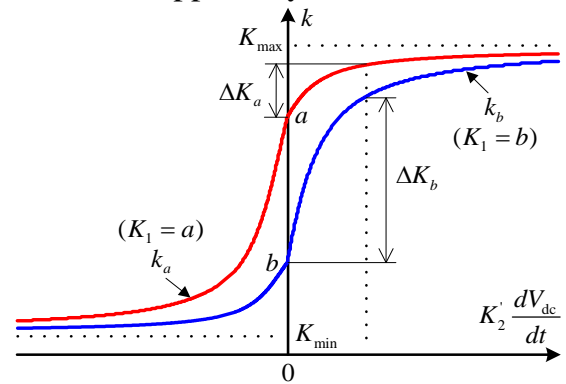

Fig. 7. Improved variable droop coefficients with different values of $K_{1}$.

Considering two different values of $K_{1}$, two improved droop coefficients are depicted in Fig. 7, where $k_{a}$ and $k_{b}$ correspond to the improved $k$ with $K_{1}=a$ and $K_{1}=b(a>b)$ respectively. A power disturbance can lead to the variation of the improved $k$. Taking $K_{2} d V_{\mathrm{dc}} / d t>0$ for example, $\Delta K_{a}$ represents the variation of $k_{a}$ while $\Delta K_{b}$ represents the variation of $k_{b}$. As $\Delta K_{a}$ is smaller than $\Delta K_{b}$ the swing amplitude of the droop curve with $k_{a}$ is smaller than that with $k_{b}$. Thus it can be concluded that the virtual inertia supplied by $\mathrm{ADC}$ with a smaller $K_{1}$ is larger. Similarly, the virtual inertia supplied by ADC with a larger $K_{1}$ is larger when $K_{2}^{\prime} d V_{\text {dc }} d t<0$.

\section{Controller Implementation}

The proposed strategy utilizes fast swing of the droop curve to release/absorb energy quickly for emulating the inertial response. Thus it can be implemented to any droop controlled converters by changing the fixed droop coefficient with a variable one. For the DC grid shown in Fig. 1, the G-VSC and E-DCT normally adopt droop control for coordinated DC voltage regulation. Both of them can participate in virtual inertial support with ADC. However, to reduce the power fluctuations of $\mathrm{AC}$ grids or the repeat adjustments of the power supply, inertial response is preferably provided by the ESS. 
However, if there is no ESS in the grid, the ADC has to be implemented in the G-VSC or other droop controlled converters. The block diagram of the proposed ADC is depicted in Fig. 8.

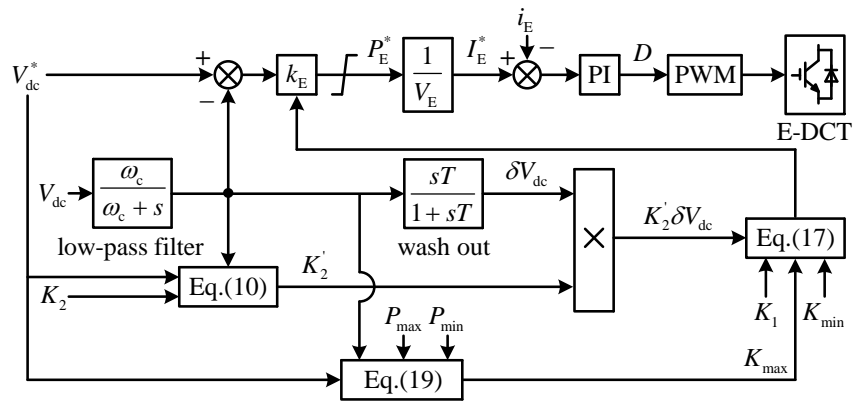

Fig. 8. Structure diagram of ADC for E-DCT.

In this paper, the proposed $\mathrm{ADC}$ is implemented on the EDCT. When a power disturbance occurs, the BESS supplies auxiliary power to slow down the change rate of the DC bus voltage to improve the DC voltage quality. Due to the inertial energy generated by the E-DCT, the initial power fluctuations within the DC grid are mostly balanced by the BESS, which leads to reduced power fluctuations of the AC grid. Compared with the battery capacity, the supplied transient auxiliary power is relatively small. Therefore, the implementation of the proposed ADC on the E-DCT has little impact on the state of charge (SOC) of the BESS.

The droop curve is designed to swing at the initial stage of disturbances to improve the inertia of DC grids, and should not be affected by the steady voltage deviation. Thus, a washout filter is adopted to achieve the transient voltage variation $\delta V_{\mathrm{dc}}$, and to reject steady state inputs. The time constant $T$ of the washout filter is mainly determined by the designed cut-off frequency of the DC voltage variation.

Normally, the inertial response is provided during the initial tens to hundreds of milliseconds under disturbances. The power/current reference for this inertial support is generated by the fast swing of the droop curve, which depends on the voltage change rate rather than the bandwidth of the outer loop. Meanwhile, the bandwidth of the inner current loop of the DCDC or AC-DC PWM converter is high enough to track the current reference variation in tens of milliseconds. After the inertial support period, the droop coefficient will recover to its origin constant value. Therefore, the bandwidth matching of the inner loop and outer loop can just follow the design principle of the conventional droop control, which makes the ADC easier to implement in droop controlled converters.

Parameters affecting ADC include $K_{1}, K_{2}, K_{\min }$ and $K_{\max }$, as can be seen in (17). For a certain DC grid, the value $K_{1}$ is fixed to meet the requirements of the primary voltage regulation. As analyzed above, $K_{\min }$ is set to zero and $K_{\max }$ is calculated in real time according to the operation state. Therefore, the virtual inertia coefficient $K_{2}$ becomes the key controllable parameter to regulate the virtual inertia supplied by ADC. Equation (13) reveals that with a larger $K_{2}$, both the virtual capacitance and supplied virtual inertia become larger. However, excessively large inertia would result in increased power demand for the converter doing inertia control, and slowing down the voltage recovery process. Therefore, how much virtual inertia should be supplied to DC grid depends on the requirements to voltage quality and system stability.

\section{Stability ANAlysis of The PROPOSED ADC STRATEGY}

Voltage stability has drawn increased attentions in DC systems due to the high penetration of power electronic converters [26-27]. The virtual capacitance can improve the voltage quality of DC grids by suppressing voltage mutation rate. However, very large virtual capacitance may lead to system instability under a very low voltage change rate. In order to investigate the stability of the ADC, the small-signal model of a DC grid is established and its eigenvalue analysis is provided.

\section{A. Small-signal modelling of the DC grid}

\section{1) Modelling of ESS terminal}

The adaptive droop control is implemented on the ESS side converter E-DCT. Taking $V_{\mathrm{dc}}<V_{\mathrm{dc}}^{*}$ and $\delta V_{\mathrm{dc}}<0$ as an example, the droop coefficient $k_{\mathrm{E}}$ for E-DCT can be expressed as

$$
k_{\mathrm{E}}=K_{1}+\frac{P_{\mathrm{max}} /\left(V_{\mathrm{dc}}^{*}-V_{\mathrm{dc}}\right)-K_{1}}{\pi / 2} \arctan \left(-K_{2} \delta V_{\mathrm{dc}}\right)
$$

The duty cycle of the DC-DC converter is regulated by a PI controller to track the current reference generated by the ADC. The control diagram of the E-DCT including the low-pass and washout filters for small-signal stability analysis is shown in Fig. 9, where $\varphi$ and $\theta$ are intermediate variables and $\omega_{\mathrm{c}}$ is the cut-off frequency of the low-pass filter.

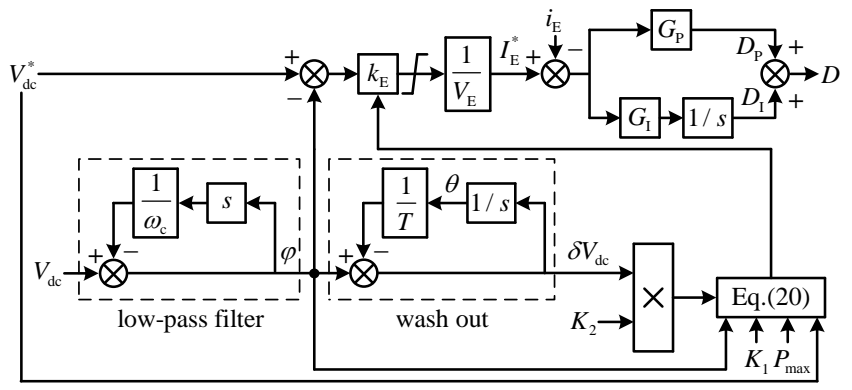

Fig. 9. Control diagram of the E-DCT for small-signal stability analysis.

The dynamic model of the E-DCT is given as

$$
\left\{\begin{array}{l}
L_{\mathrm{E}} \frac{d i_{\mathrm{E}}}{d t}=V_{\mathrm{E}}-\left(1-D_{\mathrm{P}}-D_{\mathrm{I}}\right) V_{\mathrm{dc}} \\
\frac{d D_{\mathrm{I}}}{d t}=G_{\mathrm{I}}\left(k_{\mathrm{E}} \frac{V_{\mathrm{dc}}^{*}-\varphi}{V_{\mathrm{E}}}-i_{\mathrm{E}}\right)
\end{array}\right.
$$

where $L_{\mathrm{E}}$ is the inductance of the E-DCT. $i_{\mathrm{E}}$ and $V_{\mathrm{E}}$ are the current and voltage of the BESS. $D_{\mathrm{P}}$ and $D_{\mathrm{I}}$ are the duty cycles calculated from the proportional and integral control parts respectively. $G_{\mathrm{I}}$ is the integral coefficient of the PI controller. $k_{\mathrm{E}}$ is the droop coefficient of the ADC.

By using small signal approximation, (22) is linearized as

$$
\frac{d \Delta \boldsymbol{x}_{\mathrm{E}}}{d t}=\boldsymbol{A}_{\mathrm{E}} \Delta \boldsymbol{x}_{\mathrm{E}}+\boldsymbol{B}_{\mathrm{E}} \Delta \boldsymbol{y}
$$

where $\Delta \boldsymbol{x}_{\mathrm{E}}=\left[\begin{array}{ll}\Delta i_{\mathrm{E}} & \Delta D_{\mathrm{I}}\end{array}\right]^{\mathrm{T}}, \Delta \boldsymbol{y}=\left[\begin{array}{lll}\Delta V_{\mathrm{dc}} & \Delta \varphi & \Delta \theta\end{array}\right]^{\mathrm{T}}$. Matrixes $\boldsymbol{A}_{\mathrm{E}}$ and $\boldsymbol{B}_{\mathrm{E}}$ are given in Appendix.

\section{2) Modelling of the DC grid}

In order to simplify the small-signal analysis process, the following assumptions are given. The AC grid terminal is assumed as to be an infinite power source connected by an ideal 
converter with droop control. The DC line impedance is neglected, and the PV terminal and wind turbine terminal are simply equivalent to power sources. All terminals are assumed to be connected to the same DC bus. Thus, according to Kirchhoff's current law (KCL), the dynamic model of the DC link is given as

$$
C_{\mathrm{dc}} \frac{d V_{\mathrm{dc}}}{d t}=I_{\mathrm{dc} \_\mathrm{G}}+I_{\mathrm{dc} \_\mathrm{E}}+I_{\mathrm{dc} \_ \text {RES }}-I_{\mathrm{dc} \_\mathrm{L}}
$$

where $C_{\mathrm{dc}}$ is the total capacitance of the common DC bus. $I_{\mathrm{dc} \_\mathrm{G}}$, $I_{\mathrm{dc} \_\mathrm{E}}, I_{\mathrm{dc} \_ \text {RES }}$ and $I_{\mathrm{dc} \_\mathrm{L}}$ are the respective DC side currents of the AC grid terminal, ESS terminal, RES terminal and load terminal, and can be further expressed as

$$
\left\{\begin{array}{l}
I_{\mathrm{dc}_{-} \mathrm{G}}=k_{\mathrm{G}}\left(V_{\mathrm{dc}}^{*}-V_{\mathrm{dc}}\right) / V_{\mathrm{dc}} \\
I_{\mathrm{dc} \_\mathrm{E}}=\left(1-D_{\mathrm{P}}-D_{\mathrm{I}}\right) i_{\mathrm{E}} \\
I_{\mathrm{dc} \_ \text {RES }}=P_{\mathrm{RES}} / V_{\mathrm{dc}} \\
I_{\mathrm{dc} \_\mathrm{L}}=P_{\mathrm{L}} / V_{\mathrm{dc}}
\end{array}\right.
$$

where $k_{\mathrm{G}}$ is the droop coefficient of the G-VSC. $P_{\mathrm{RES}} 、 P_{\mathrm{L}}$ are the RES power and load power respectively.

As shown in Fig. 9, the equations of $\varphi$ and $\theta$ for small-signal analysis can be expressed as

$$
\begin{gathered}
\frac{d \varphi}{d t}=\omega_{\mathrm{c}}\left(V_{\mathrm{dc}}-\varphi\right) . \\
\frac{d \theta}{d t}=\varphi-\frac{\theta}{T} .
\end{gathered}
$$

Taking $V_{\mathrm{dc}}, \varphi$ and $\theta$ as state variables, (23), (25) and (26) can be linearized as

$$
\frac{d \Delta \boldsymbol{y}}{d t}=\boldsymbol{Y}_{\mathrm{E}} \Delta \boldsymbol{x}_{\mathrm{E}}+\boldsymbol{Y}_{y} \Delta \boldsymbol{y}
$$

where matrixes $\boldsymbol{Y}_{\mathrm{E}}$ and $\boldsymbol{Y}_{\mathrm{y}}$ are given in Appendix.

According to (22) and (27), the linearized small-signal model of the DC grid is given as

$$
\frac{d \Delta \boldsymbol{x}}{d t}=\boldsymbol{A}_{\mathrm{sys}} \Delta \boldsymbol{x}
$$

where $\Delta \boldsymbol{x}=\left[\begin{array}{ll}\Delta x_{\mathrm{E}} & \Delta y\end{array}\right]^{\mathrm{T}}$, and the complete matrix $\boldsymbol{A}_{\mathrm{sys}}$ is given in Appendix.

\section{B. Small-Signal Stability Analysis}

The parameters and initial conditions of the DC grid are given in Table AI in Appendix. Based on the small-signal model expressed by (28), the trajectories of the eigenvalues with the virtual inertia coefficients $K_{2}$ varying from 50 to 5500 in step of 50 are shown in Fig. 10. In this case, the control parameters of ADC and load conditions are set as: $f_{\mathrm{c}}=200 \mathrm{~Hz}$, $T=0.1 \mathrm{~s}, G_{\mathrm{P}}=2, G_{\mathrm{I}}=50, P_{\mathrm{L}}=25 \mathrm{~kW}, P_{\mathrm{RES}}=10 \mathrm{~kW}$. It can be seen that, with the increase of the virtual inertia coefficient $K_{2}$, the real parts of the eigenvalues approach to zero, and become positive for $K_{2}>5200$. Therefore, $K_{2 \_\max }=5200$ is considered to be the upper limit of the variable droop coefficient in the system stable region under the above condition.

However, the load/DG power changes and control parameters variations may affect the system stability with ADC. Hence system stability using eigenvalue analysis with different load conditions and control parameters is further investigated to determine $K_{2 \_ \text {max. }}$ The upper limits of $K_{2}$ under different load/DG power and control parameters are given in Table I and
Table II respectively. It can be seen that, to guarantee system stability, the maximum allowed $K_{2}$ (i.e. $K_{2 \_ \text {max }}$ ) varies during load variation, and the increasing of $T$ and $G_{\mathrm{I}}$ or the decreasing

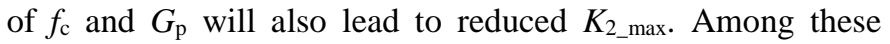
results, the minimum $K_{2 \_ \text {max }}$ is 1700 within reasonable variations of the control parameters. Therefore, $k_{2}$ max is set to smaller than 1000 to ensure system stability while still providing adequate inertial support for the DC grid. Taking $K_{2}$ $=500$ as an example, and the root locus with control parameters varying of $f_{\mathrm{c}}(50 \sim 1000 \mathrm{~Hz}), T(0.01 \sim 0.5 \mathrm{~s}), G_{\mathrm{P}}(0.5 \sim 5)$ and $G_{\mathrm{I}}$ $(5 \sim 150)$ are analyzed and shown in Figs. 11 (a)-(d) respectively. As can be seen, all the eigenvalues are on the left half-plane during the control parameters variation. Thus, the system stability can be guaranteed when a lower $K_{2}\left(\right.$ e.g. $\left.K_{2}<1000\right)$ is adopted.

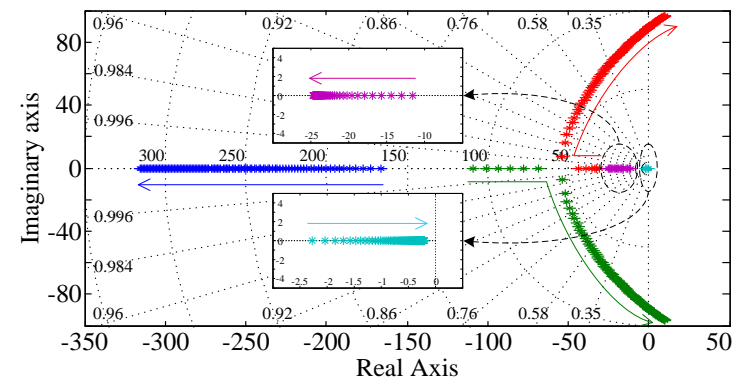

Fig. 10. The root locus of the ADC for the DC grid with different $K_{2}$.

TABLE I

Maximum Values of $K_{2}$ under Different Operating Conditions

\begin{tabular}{cc||cc||cc}
\hline$P_{\mathrm{L}}-P_{\mathrm{RES}}$ & $K_{2 \_\max }$ & $P_{\mathrm{L}}-P_{\mathrm{RES}}$ & $K_{2 \_\max }$ & $P_{\mathrm{L}}-P_{\mathrm{RES}}$ & $K_{2 \_\max }$ \\
\hline $5 \mathrm{~kW}$ & 8950 & $15 \mathrm{~kW}$ & 5200 & $25 \mathrm{~kW}$ & 4900 \\
$10 \mathrm{~kW}$ & 6250 & $20 \mathrm{~kW}$ & 4850 & $30 \mathrm{~kW}$ & 5500 \\
\hline
\end{tabular}

TABLE II

Maximum Values of $K_{2}$ under Different Control Parameters

\begin{tabular}{cc||cc||cc||cc}
\hline$f_{\mathrm{c}}$ & $K_{2 \_\max }$ & $T$ & $K_{2 \_\max }$ & $G_{\mathrm{P}}$ & $K_{2 \_\max }$ & $G_{\mathrm{I}}$ & $K_{2 \_\max }$ \\
\hline $50 \mathrm{~Hz}$ & 4150 & $0.01 \mathrm{~s}$ & 7650 & 0.5 & 1700 & 5 & 5500 \\
$100 \mathrm{~Hz}$ & 4500 & $0.05 \mathrm{~s}$ & 5150 & 1 & 3450 & 20 & 5250 \\
$200 \mathrm{~Hz}$ & 4850 & $0.1 \mathrm{~s}$ & 4850 & 2 & 4850 & 50 & 4850 \\
$500 \mathrm{~Hz}$ & 5150 & $0.2 \mathrm{~s}$ & 4700 & 3 & 5200 & 100 & 4250 \\
$1000 \mathrm{~Hz}$ & 5400 & $0.5 \mathrm{~s}$ & 4600 & 5 & 5450 & 150 & 3850 \\
\hline
\end{tabular}
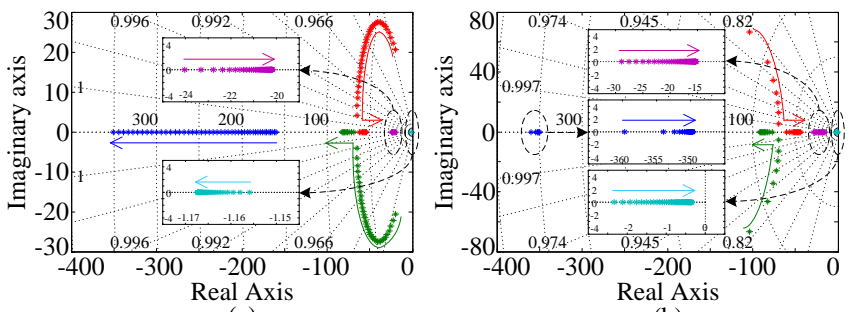

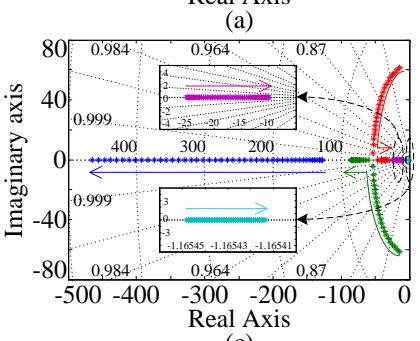

(c)

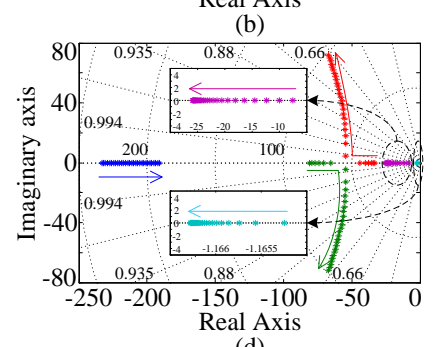

(d)
Fig. 11. The root locus with control parameters varying. (a) Varying of $f_{\mathrm{c}}$. (b) Varying of $T$. (c) Varying of $G_{\mathrm{P}}$. (d) Varying of $G_{\mathrm{I}}$. 
Under the premise of system stability, an adjustable virtual capacitance can be achieved by changing the droop coefficient in a large extent $\left(0<K_{2}<1000\right)$, which can smooth and damp the higher frequency oscillations. Furthermore, the voltage change rate can be reduced effectively by the virtual capacitance. This would benefit the fault ride through (FRT) of the converters in DC grids under an abrupt disturbance, due to achieve a longer duration for FRT. Therefore, the proposed strategy improves the high frequency stability.

Since the inertial response by the proposed ADC is only emulated during the initial tens to hundreds of milliseconds of disturbances, it can effectively suppress voltage oscillations in the frequency range of several $\mathrm{Hz}$ to tens of $\mathrm{Hz}$, which is called higher-frequency oscillations in this paper. For low frequency disturbances, some recent studies have shown that droop controlled converters may affect low-frequency stability of DC grids [27]. However, the virtual capacitance cannot be set too large considering the system stability. Moreover, in order to avoid the influence on the primary voltage regulation and power sharing of the droop control, the washout function is designed to filter out DC voltage deviation and low frequency voltage fluctuations below $1 \mathrm{~Hz}$. Therefore, the proposed ADC can be regarded as the conventional droop control under steady state and low frequency power/DC voltage variation.

\section{HARDWARE-IN-THE-LOOP TESTING RESULTS}

Experimental tests on a controller hardware in-the-loop simulation platform of a LV DC grid have been carried out to validate the proposed control strategy. The HIL platform shown in Fig. 12 is composed of a real-time digital simulator (an OP5600 RT-LAB), a TI's TMS320F28335 DSP board and a host computer. As illustrated in Fig. 12(a), control algorithms containing the proposed ADC for the BESS are deployed in DSP, while the control algorithms for other terminals and the real-time simulation model of a low-voltage DC grid are deployed in RT-LAB. The PWM pulses generated by DSP for E-DCT are fed to the pulse input port of RT-LAB through an optoelectronic isolation module, as shown in Fig. 12(b). The min-BNC interfaces of RT-LAB are connected with a DL850 wave recorder to display the test results. Table AI in Appendix lists the detailed parameters of the real-time simulation model illustrated in Fig. 12(a), where $P_{\mathrm{WT}}, P_{\mathrm{L}}, P_{\mathrm{B}}$ and $P_{\mathrm{G}}$ are the PMSG-based wind turbine output power, AC loads power,
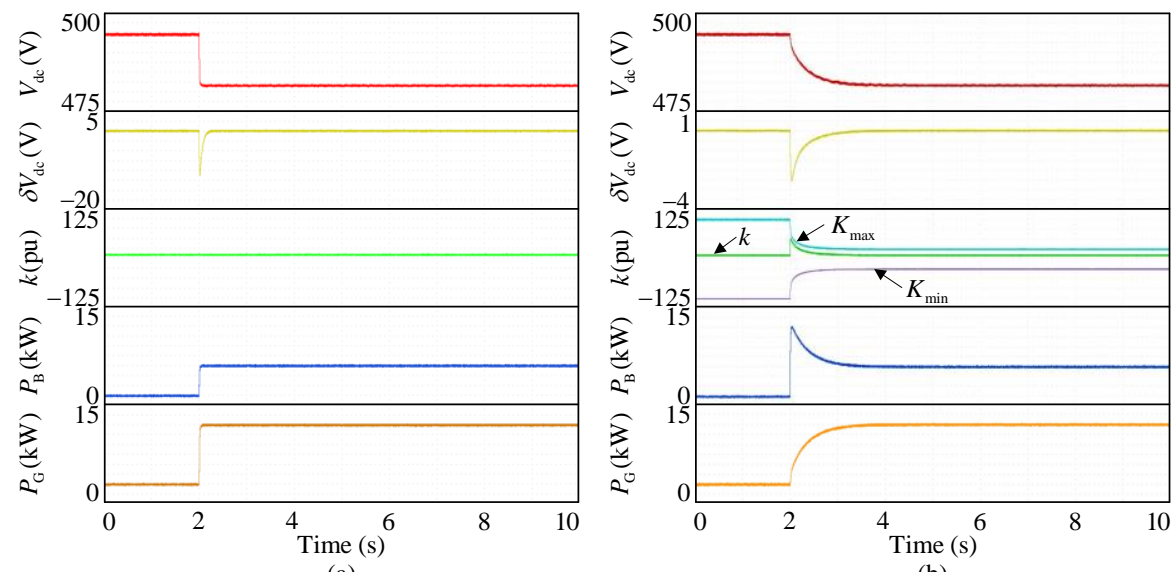

(a)
BESS power and AC grid power, respectively.

In this paper, the G-VSC and E-DCT are coordinated by voltage droop control and the proposed ADC is implemented on E-DCT. The control parameters for G-VSC and E-DCT are also listed in Table AI. Meanwhile, $K_{\min }$ is set to zero and $K_{\max }$ is calculated in real time, while different values of $K_{2}$ can be selected to acquire different virtual inertia responses. For the experimental results, positive $P_{\mathrm{G}}$ and $P_{\mathrm{B}}$ refer to the AC grid and BESS output power to the DC grid. $k$ is the droop coefficient for E-DCT. $P_{\mathrm{R}}$ is the required power of the LV DC grid caused by the wind turbine and the AC loads.

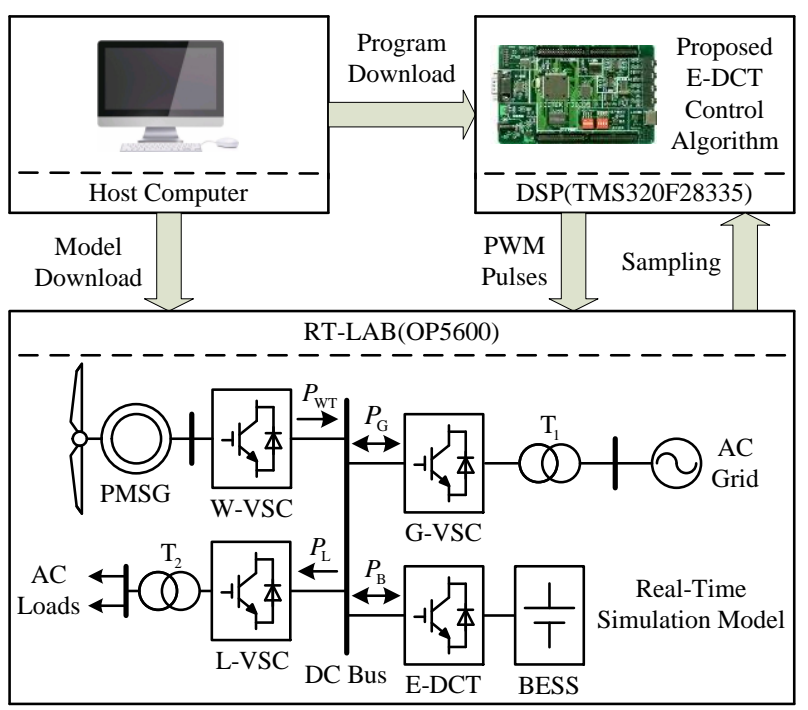

(a)

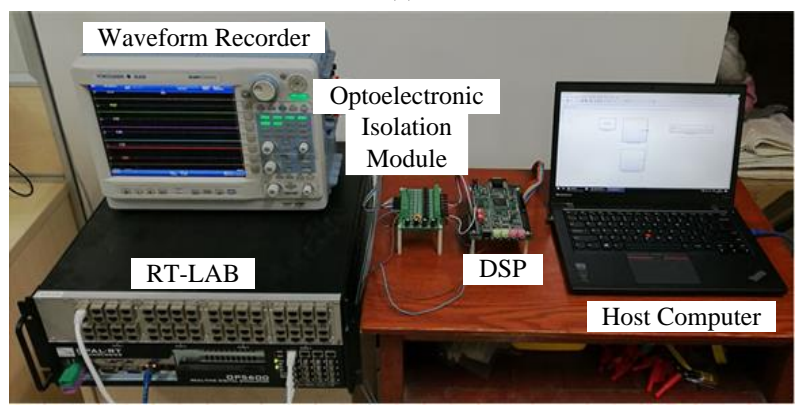

(b)

Fig. 12. HILS platform of a DC grid. (a) System design. (b) Experimental platform.

Fig. 13. Dynamic responses during sudden power increase. (a) Without virtual inertia control. (b) With $\mathrm{ADC}\left(K_{2}=500\right)$. (c) With $\mathrm{ADC}\left(K_{2}=3000\right)$. 

and Electronic Engineering Copyright. The copy of record is available at IEEE Xplore Digital Library.

\section{A. Sudden Power Change Test}

In order to verify the proposed ADC and analyze the impact caused by different values of $K_{2}$, tests under sudden power changes are performed firstly. In Fig. 13, the required power is increased from $4.5 \mathrm{~kW}$ to $18 \mathrm{~kW}$, while in Fig. 14, the required power is decreased from $18 \mathrm{~kW}$ to $4.5 \mathrm{~kW}$.

Fig. 13(a) shows the test results without virtual inertia control. At the beginning, the DC bus voltage $V_{\mathrm{dc}}$ is controlled around $495 \mathrm{~V}$ by E-DCT and G-VSC. At $2 \mathrm{~s}$, the required power suddenly increases, and due to the small system inertia, $V_{\mathrm{dc}}$ quickly droops to $480 \mathrm{~V}$ and a large voltage variation $\delta V_{\mathrm{dc}}$ of $11 \mathrm{~V}$ can be observed. Based on the same condition, Fig. 13(b) shows the test results when the proposed ADC with $K_{2}=500$ is applied. As can be seen, the droop coefficient $k$ for E-DCT varies with $V_{\mathrm{dc}}$ and the auxiliary power is supplied by the BESS to enhance the system inertia. After the sudden power increase, $k$ is increased from $10 \mathrm{pu}$ to $50 \mathrm{pu}$ and $P_{\mathrm{B}}$ is increased to supply the auxiliary power of $6 \mathrm{~kW}$. As a result, a reduced $\delta V_{\mathrm{dc}}$ of $2.5 \mathrm{~V}$ is observed. Due to the virtual inertia, the transient time of $V_{\mathrm{dc}}$ is prolonged, and the change of $P_{\mathrm{G}}$ is slowed down. To supply larger virtual inertia, ADC with $K_{2}=3000$ is applied and the test results are demonstrated in Fig. 13(c). When the required power suddenly increases, $k$ is increased to $75 \mathrm{pu}$ and $P_{\mathrm{B}}$ is increased to supply a larger auxiliary power of $8 \mathrm{~kW}$. As a result, a smaller $\delta V_{\mathrm{dc}}$ of $-1 \mathrm{~V}$ is observed and the transient time is much prolonged. The comparison between Fig. 13(b) and Fig. 13(c) reveals that with a larger $K_{2}$, the virtual inertia supplied by ADC is larger.

Fig. 14 shows the test results under a sudden power decrease, and the measurement noise is added to the DC voltage signal to replicate practical conditions. As can be seen from the filtered voltage, i.e. $V_{\mathrm{dc}}$, the measurement noise is almost eliminated by the low-pass filter with a cut-off frequency of $200 \mathrm{~Hz}$. Thus, the $\delta V_{\mathrm{dc}}$ can still properly reflect the change rate of the DC voltage despite introducing the noise interference. From Fig. 13 and Fig. 14 , it can be seen that the limits are calculated in real-time and $k$ adaptively varies within the limits. Furthermore, the virtual inertia supplied by ADC can be regulated by adjusting the value of $K_{2}$, so different requirements for virtual inertia can be met.

\section{B. Higher-frequency Random Power Fluctuations Test}

To further illustrate the advantages of the proposed ADC, tests of higher-frequency random power fluctuations are carried out and the results are shown in Fig. 15. At the beginning, the required power $P_{\mathrm{R}}$ of the DC grid fluctuates between $4.5 \mathrm{~kW}$ and $9 \mathrm{~kW}$. At $5 \mathrm{~s}$, a load of $9 \mathrm{~kW}$ is switched on.

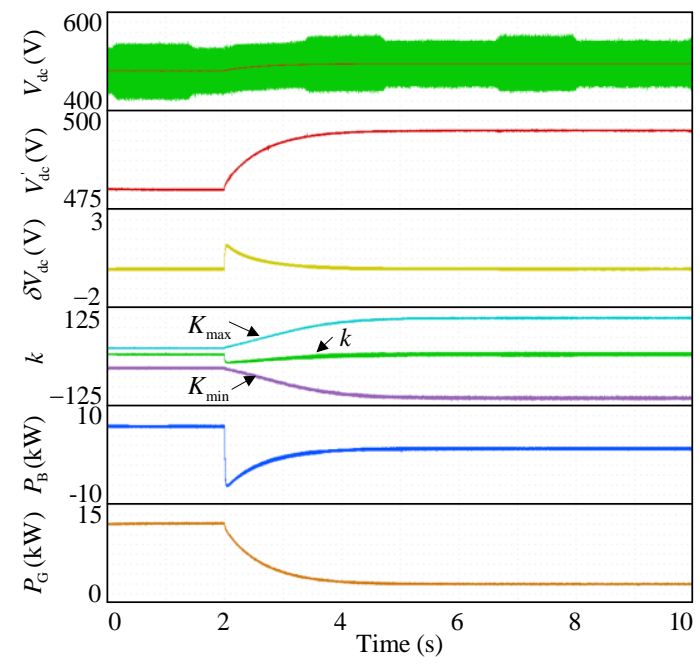

Fig. 14. Dynamic responses with $\operatorname{ADC}\left(K_{2}=500\right)$ during sudden power decrease while considering the measurement noise.

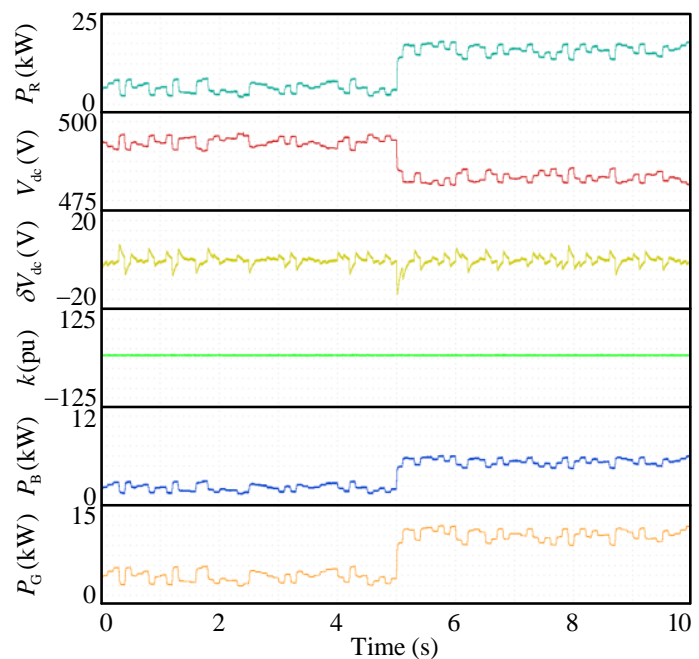

(a)

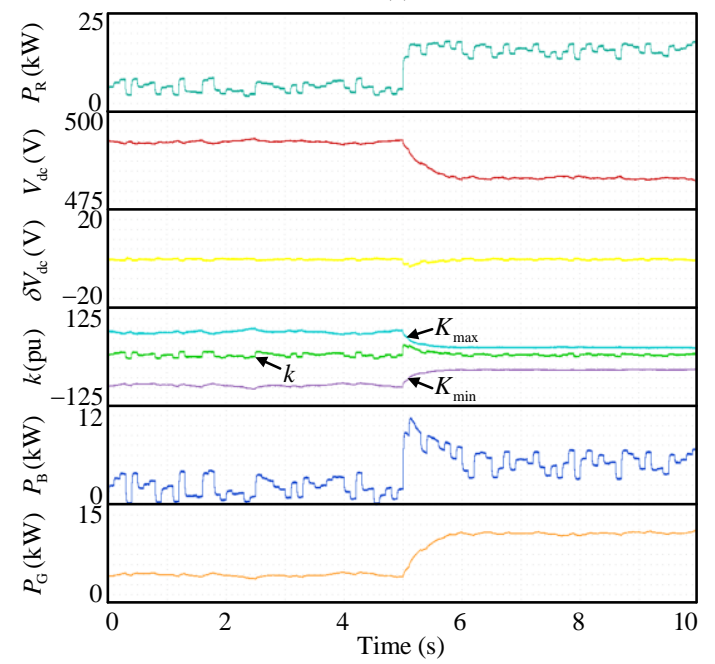

(b)

Fig. 15. Dynamic responses during random power fluctuations. (a) Without virtual inertia control. (b) With ADC.

Fig. 15(a) shows the system dynamic responses without virtual inertia control. Due to the lack of inherent inertia, $V_{\mathrm{dc}}$ changes significantly. Meanwhile, the power fluctuations within the DC grid are transmitted to the AC grid, which is not 
desirable for the AC grid. Fig. 15(b) shows the test results when the proposed ADC with $K_{2}=500$ is applied. It can be seen that $V_{\mathrm{dc}}$ is significantly smoothed due to the virtual inertia. As the power fluctuations are mostly balanced by the BESS, a much smoother $P_{\mathrm{G}}$ is also observed. Auxiliary power is supplied by the BESS and the average value of $P_{\mathrm{B}}$ is almost the same as that in Fig. 15(a), so the implementation of ADC has little impact on the SOC of the BESS.

The virtual capacitance only suppresses voltage flicker and high frequency variation, without affecting the steady-state voltage deviation. Fig. 13 shows the reduction of the voltage change rate using the ADC in the event of the load step changes, which can help to reduce the voltage flicker. Fig. 15 shows the high frequency variations are suppressed, which can help to smooth the DC bus voltage and enhance voltage quality. The voltage deviation still exits due to droop control, but it can be further reduced and even restored to the rated value by the secondary voltage regulation. The results of this voltage recovery process are not given due to the secondary voltage regulation is not studied in this paper.

\section{Coordination of Two Terminals with ADC}

In this case, both the AC grid and ESS terminals adopt ADC to investigate their interaction with their virtual inertia coefficients set as $K_{2 \mathrm{G}}=K_{2 \mathrm{E}}=500$. In addition, the system performances under lower-frequency $(0.2 \mathrm{~Hz})$ and higherfrequency $(20 \mathrm{~Hz})$ oscillations caused by the load fluctuations are incorporated in the case studies. The HIL test results are shown in Fig. 16. As can be seen, the ADC can work properly for both oscillations, but the damping effect of the higherfrequency oscillation is obviously better than that of lowfrequency oscillation. Due to both AC grid terminal and ESS terminal provide inertial responses, the power oscillations caused by the load variation are shared by them according to their capacity.

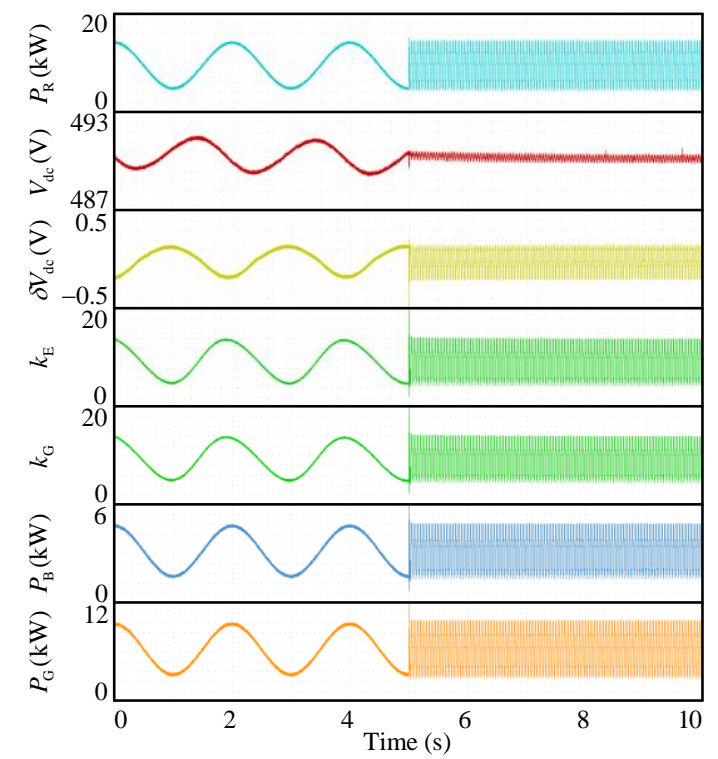

Fig. 16. Dynamic responses with multiple ADC during lower- and higherfrequency oscillations.

\section{CONCLUSION}

Under the fixed droop control, the distinct unbalanced power added to the small capacitor at the initial stage of the disturbances can result in rapid changes of the DC voltage. This paper proposes an adaptive droop control strategy to increase the equivalent inertia of the DC grid. It can not only mitigate the mutation rate and fluctuations of the DC voltage and improve the power supply quality, but also has the following advantages.

(1) By simply replacing the fixed droop coefficient with the adaptive one, the ADC can be easily applied to droop controlled converters without adding any auxiliary controller or observer. The emulated virtual capacitance can be adjusted flexibly by changing the virtual inertia coefficient $K_{2}$, to meet the requirements of voltage quality while ensuring system stability.

(2) It can provide both inertial response and primary voltage regulation for the DC grid in an integrated controller. The ADC strategy can make the droop curve swing quickly during the initial stage of large disturbances to provide inertial support, and recover to the conventional droop control as the voltage gradually becomes stable. By introducing the arc-tangent function, the ADC properly swings the droop curve to achieve a larger inertia without causing system oscillation. Meanwhile, the real-time calculation of the limits ensures the ADC adaptable to different operation states within the allowed range.

(3) It has negligible effect on power sharing among the converters in the DC grid and power quality in the AC grid. The converter with ADC only provides additional transient power support, and has constant droop coefficient during the subsequent power distribution by the system primary and secondary regulation. Considering that power fluctuations are not desirable for the AC grid, the proposed ADC implemented on the BESS can also smooth the AC grid power exchange to improve the power quality of the AC grid.

\section{APPENDIX}

The complete matrixes of the DC grid small-signal model are given below.

$$
\begin{aligned}
& \boldsymbol{A}_{\mathrm{sys}}=\left[\begin{array}{ll}
\boldsymbol{A}_{\mathrm{E}} & \boldsymbol{B}_{\mathrm{E}} \\
\boldsymbol{Y}_{\mathrm{E}} & \boldsymbol{Y}_{y}
\end{array}\right] \\
& \boldsymbol{A}_{\mathrm{E}}=\left[\begin{array}{cc}
-\frac{G_{\mathrm{P}} V_{\mathrm{dc} 0}}{L_{\mathrm{E}}} & \frac{V_{\mathrm{dc} 0}}{L_{\mathrm{E}}} \\
-G_{\mathrm{I}} & 0
\end{array}\right] \\
& \boldsymbol{B}_{\mathrm{E}}=\left[\begin{array}{ccc}
\frac{-1+D_{0}}{L_{\mathrm{E}}} & \frac{\mu G_{\mathrm{P}} V_{\mathrm{dc} 0}}{L_{\mathrm{E}}} & \frac{\lambda G_{\mathrm{P}} V_{\mathrm{dc} 0}}{L_{\mathrm{E}}} \\
0 & \mu G_{\mathrm{I}} & \lambda G_{\mathrm{I}}
\end{array}\right] \\
& \boldsymbol{Y}_{\mathrm{E}}=\left[\begin{array}{cc}
\frac{1-D_{0}+G_{\mathrm{P}} i_{\mathrm{E} 0}}{C_{\mathrm{dc}}} & -\frac{i_{\mathrm{E} 0}}{C_{\mathrm{dc}}} \\
0 & 0 \\
0 & 0
\end{array}\right]
\end{aligned}
$$



and Electronic Engineering Copyright. The copy of record is available at IEEE Xplore Digital Library.

$$
\boldsymbol{Y}_{\mathrm{y}}=\left[\begin{array}{ccc}
-\frac{k_{\mathrm{G}} V_{\mathrm{dc}}^{*}+P_{\mathrm{RES}}-P_{\mathrm{L}}}{C_{\mathrm{dc}} V_{\mathrm{dc} 0}^{2}} & -\frac{\mu G_{\mathrm{P}} i_{\mathrm{E} 0}}{C_{\mathrm{dc}}} & -\frac{\lambda G_{\mathrm{P}} i_{\mathrm{E} 0}}{C_{\mathrm{dc}}} \\
\omega_{\mathrm{c}} & -\omega_{\mathrm{c}} & 0 \\
0 & 1 & -\frac{1}{T}
\end{array}\right]
$$

[6] N. Chaudhuri, and B. Chaudhuri, "Adaptive droop control for effective power sharing in multi-terminal DC Microgrids," IEEE Trans. Power Syst., vol. 28, no. 1, pp. 21-29, Feb. 2013.

[7] C. Gavriluta, I. Candela, A. Luna, A. Gomez-Exposito, and P. Rodriguez, "Hierarchical control of HV-MTDC systems with droop-based primary and OPF-based secondary," IEEE Trans. Smart Grid., vol. 6, no. 3, pp. 1502-1510, May 2015.

[8] P.-K. Keung, P. Li, H. Banakar, and B. T. Ooi, "Kinetic energy of windturbine generators for system frequency support," IEEE Trans. Power Syst., vol. 24, no. 1, pp. 279- 287, Feb. 2009.

where the coefficients $\mu$ and $\lambda$ in the matrixes are expressed as

$$
\begin{gathered}
\mu=\frac{V_{\mathrm{dc}}^{*}-\varphi_{0}}{V_{\mathrm{E}}} \alpha-\frac{k_{\mathrm{E} 0}}{V_{\mathrm{E}}} \\
\lambda=\frac{V_{\mathrm{dc}}^{*}-\varphi_{0}}{V_{\mathrm{E}}} \beta \\
\alpha=\frac{2}{\pi} \frac{1}{1+K_{2}^{2}\left(\varphi_{0}-\theta_{0} / T\right)^{2}}\left(K_{1}+\frac{P_{\max }}{\varphi_{0}-V_{\mathrm{dc}}^{*}}\right) K_{2}- \\
\frac{2}{\pi} \frac{P_{\max }}{\left(\varphi_{0}-V_{\mathrm{dc}}^{*}\right)^{2}} \arctan \left(K_{2} \varphi_{0}-\frac{K_{2}}{T} \theta_{0}\right) \\
\beta=-\frac{2}{\pi} \frac{1}{1+K_{2}^{2}\left(\varphi_{0}-\theta_{0} / T\right)^{2}}\left(K_{1}+\frac{P_{\max }}{\varphi_{0}-V_{\mathrm{dc}}^{*}}\right) \frac{K_{2}}{T} .
\end{gathered}
$$

\begin{tabular}{|c|c|}
\hline Items & Parameters \& Values \\
\hline DC Bus Voltage & $V_{\mathrm{dcN}}=500 \mathrm{~V}$ \\
\hline AC Grid & $\begin{array}{l}S_{\mathrm{N}}=33 \mathrm{kVA}, V_{\mathrm{acN}}=380 \mathrm{~V}, L_{\mathrm{s}}=0.05 \mathrm{pu} \\
\mathrm{T} 1: k_{\mathrm{T} 1}=380 / 220, L_{\mathrm{T} 1}=0.05 \mathrm{pu}\end{array}$ \\
\hline G-VSC (Two-level VSC) & $\begin{array}{l}P_{\mathrm{N}}=30 \mathrm{~kW}, V_{\mathrm{N}}=220 \mathrm{~V}, C=2000 \mu \mathrm{F} \\
\text { Droop controller: } V_{\mathrm{dc}}^{*}=500 \mathrm{~V}, P_{\min }=- \\
30 \mathrm{~kW}, P_{\max }=30 \mathrm{~kW}, k_{\mathrm{G}}=10 \\
\text { Current controller (p.u.): } k_{\mathrm{p}}=1 \quad k_{\mathrm{l}}=50\end{array}$ \\
\hline BESS & $300 \mathrm{~V} / 200 \mathrm{~A} \cdot \mathrm{h}$ \\
\hline $\begin{array}{l}\text { E-DCT (Bi-direction DC- } \\
\text { DC converter) }\end{array}$ & $\begin{array}{l}P_{\mathrm{N}}=15 \mathrm{~kW}, V_{\mathrm{iN}}=300 \mathrm{~V}, V_{\mathrm{oN}}=500 \mathrm{~V} \\
L=1 \mathrm{mH}, C=390 \mu \mathrm{F} \\
\text { ADC: } V_{\mathrm{dc}}^{*}=500 \mathrm{~V}, P_{\max }=15 \mathrm{~kW}, K_{1}=10\end{array}$ \\
\hline PMSG & $P_{\mathrm{N}}=20 \mathrm{~kW}, v_{\mathrm{N}}=12 \mathrm{~m} / \mathrm{s}$ \\
\hline AC Loads & $P_{\mathrm{N}}=30 \mathrm{~kW}$ \\
\hline Initial Condition & $\begin{array}{l}k_{\mathrm{E} 0}=10, \varphi_{0}=V_{\mathrm{dc} 0}, \theta_{0}=T \cdot V_{\mathrm{dc} 0}, i_{\mathrm{E} 0}=K_{1}\left(V_{\mathrm{dc}}^{*}\right. \\
\left.V_{\mathrm{dcc}}^{*}\right) / V_{\mathrm{E}}, D_{0}=1-V_{\mathrm{E}} / V_{\mathrm{dc} 0}\left(V_{\mathrm{dc} 0} \text { is related to }\right. \\
\left.P_{\mathrm{RES}} \text { and } P_{\mathrm{L}}\right)\end{array}$ \\
\hline
\end{tabular}

The nominal parameters of the simulated DC grid and the initial condition for its small-signal analysis are given below.

TABLE AI

SYSTEM PARAMETERS AND INITIAL VALUES OF THE DC GRID

\section{REFERENCES}

[1] M. Ranjram, and P. W. Lehn, "A multiport power-flow controller for DC transmission grids," IEEE Trans. Power Del., vol. 31, no. 1, pp. 389-396, Feb. 2016.

[2] K. A. Saleh, A. Hooshyar and E. F. El-Saadany, "Hybrid passiveovercurrent relay for detection of faults in low-voltage DC Microgrids," IEEE Trans. Smart Grid, vol. 8, no. 3, pp. 1129-1138, May 2017.

[3] L. Xu and D. Chen, "Control and operation of a DC microgrid with variable generation and energy storage," IEEE Trans. Power Del., vol. 26, no. 4, pp. 2513-2522, Oct. 2011.

[4] R. Ahmadi, and M. Ferdowsi, "Improving the performance of a line regulating converter in a converter-dominated DC microgrid system," IEEE Trans. Smart Grid, vol. 5, no. 5, pp. 2553-2563, Sep. 2014.

[5] B. Liu, F. Zhuo, Y. Zhu, and H. Yi, "System operation and energy management of a renewable energy-based DC micro-grid for high penetration depth application," IEEE Trans. Smart Grid, vol. 6, no. 3, pp. 1147-1155, May 2015.
[9] X. Liu, P. Wang, and P. C. Loh, "A hybrid AC/DC microgrid and its coordination control," IEEE Trans. Smart Grid, vol. 2, no. 2, pp. 278286, Jun. 2011.

[10] H. P. Beck and R. Hesse, "Virtual synchronous machine" in 9th International Conference on Electrical Power Quality and Utilisation, Barcelona, Spain, 2007, pp. 1-6.

[11] Q.-C. Zhong and G. Weiss, "Synchronverters: Inverters that mimic synchronous generators," IEEE Trans. Ind. Electron., vol. 58, no. 4, pp. 1259-1267, Apr. 2011.

[12] Q.-C. Zhong, P.-L. Nguyen, Z. Ma, and W. Sheng, "Self-synchronized synchronverters: Inverters without a dedicated synchronization unit," IEEE Trans. Power Electron., vol. 29, no. 2, pp. 617-630, Feb. 2014.

[13] Y. Wang, J. Meng, X. Zhang, and L. X, "Control of PMSG-based wind turbines for system inertial response and power oscillation damping," IEEE Trans. Sustain. Energy, vol. 6, no. 2, pp. 565-574, Apr. 2015.

[14] B. Liu, F. Zhuo, and X. Bao, "Control method of the transient compensation process of a hybrid energy storage system based on battery and ultra-capacitor in micro-grid," in Proc. IEEE ISIE Conf, May 2012, pp. 1325-1329.

[15] D. Wu, F. Tang, T. Dragicevic, J. M. Guerrero, and J. C. Vasquez, "Coordinated control based on bus-signaling and virtual inertia for islanded DC microgrids," IEEE Trans. Smart Grid, vol. 6, no. 6, pp. 2627-2638, Nov. 2015.

[16] X. Zhu, J. Cai, Q. Yan, J. Chen, and X. Wang, "Virtual inertia control of wind-battery-based islanded DC micro-grid," in Proc. IET RPG Conf., Oct. 2015, pp. 1-6. M. Guerrero, "A virtual inertia control strategy for DC microgrids analogized with virtual synchronous machines," IEEE Trans. Ind. Electron., vol. 64, no. 7, pp. 6005-6016, Jul. 2017.

[18] E. Prieto-Araujo, A. Egea-Alvarez, S. Fekriasl, and O. Gomis-Bellmunt, "DC voltage droop control design for multiterminal HVDC systems considering AC and DC grid dynamics," IEEE Trans. Power Del., vol. 31, no. 2, pp. 575-585, Apr. 2016.

[19] Y. Gu, X. Xiang, W. Li and X. He, "Mode-adaptive decentralized control for renewable DC microgrid with enhanced reliability and flexibility," IEEE Trans. Power Electron., vol. 29, no. 9, pp. 5072-5080, Sep. 2014.

[20] K. Rouzbehi, A. Miranian, J. I. Candela, A. Luna and P. Rodriguez, "A generalized voltage droop strategy for control of multiterminal DC Microgrids," IEEE. Ind. App., vol. 51, no. 1, pp. 607-618, Jan./Feb. 2015.

[21] R. Eriksson, J. Beerten, M. Ghandhari, and R. Belmans, "Optimizing DC voltage droop control settings for AC/DC system interactions," IEEE Trans. Power Del., vol. 29, no. 1, pp. 362-369, Feb. 2014.

[22] Y. Xia, W. Wei, Y. Peng, P. Yang and M. Yu, "Decentralized coordination control for parallel bidirectional power converters in a GridConnected DC Microgrid,” IEEE Trans. Smart Grid, vol. PP, no. 99, pp.1-12, Jul. 2017.

[23] Y. Xia, W. Wei, M. Yu, Y. Peng and J. Tang, "Decentralized multi-time scale power control for a hybrid AC/DC microgrid with multiple subgrids," IEEE Trans. Power Electronics, vol. PP, no. 99, pp.1-12, Jun. 2017.

[24] Y. Li, L. He, F. Liu, C. Li, Y. Cao and M. Shahidehpour, "Flexible voltage control strategy considering distributed energy storages for DC distribution network," IEEE Trans. on Smart Grid, vol. PP, no. 99, pp. 112, Jul. 2017.

[25] X. Li, L. Guo, S. Zhang, C. Wang, Y. Li, A. Chen, and Y. Feng, "Observer-based DC voltage droop and current feed-forward control of a DC microgrid,” IEEE Trans. Smart Grid, vol. PP, no. 99, pp. 1-9, Mar. 2017.

[26] M. Zhang, Y. Li, F. Liu, L. Luo, Y. Cao and M. Shahidehpour, "Voltage stability analysis and sliding mode control method for rectifier in DC systems with constant power loads," IEEE Journal of Emerging and Selected Topics in Power Electronics, vol. 5, no. 4, pp. 1621-1630, Dec. 2017.
[17] W. Wu, Y. Chen, A. Luo, L. Zhou, X. Zhou, L. Yang, Y. Dong, and J. 
This paper is a post-print of a paper submitted to and accepted for publication in IEEE Transaction on Smart Grid and is subject to Institution of Electrical and Electronic Engineering Copyright. The copy of record is available at IEEE Xplore Digital Library.

[27] N. Rashidirad, M. Hamzeh, K. Sheshyekani, and E. Afjei, “A Simplified Equivalent Model for the Analysis of Low-Frequency Stability of Multi-
Bus DC Microgrids,'IEEE Trans. Smart Grid, vol. pp, no. 99, pp. 1-1, May 2017. 